\title{
RECETA TRADICIONAL RUSSA ADAPTADA PARA DIETAS COM RESTRIÇÃO DE SÓDIO, GORDURA SATURADA E COLESTEROL
}

\author{
TRADIIONALRUSSIAN RECIPEADAPIEDFORDIEISWITHRESTRICTIONSON SODIUM, \\ SATURATEDFATANDCHOLESTEROL
}

\author{
Macarena Urresta razu DEVINC ENZ ${ }^{1}$ \\ Simone Paula MODESTO ${ }^{1}$ \\ Maria Elisabeth Machado PINTO E SILVA²
}

RESUM 0

Estudos prospectivos sobre doença isquêmica do coração detectaram fatores de risco para a doença coronariana: idade, sexo, presença de hipertensão arterial sistêmica, níveis elevados de colesterol sérico e tabagismo. Os três últimos podem ser modificados por meio de intervenções dietéticas, farmacológicas e/ou comportamentais. O potencial de uma dieta, ou de um alimento em aumentar os níveis plasmáticos de colesterol e promover aterosclerose está diretamente relacionado com seu conteúdo de colesterol e gordura saturada. Indivíduos com dietas restritas em sódio, colesterol e gordura saturada não devem consumir estrogonofe de carne, preparação tradicional russa de uso em nossa população, por conter os ingredientes: manteiga, sal e creme de leite. A fim de permitir a esses pacientes o consumo de estrogonofe e com menor teor desses nutrientes foram feitas adaptações como a substituição do creme de leite por leite desnatado e amido, da manteiga por óleo vegetal e do sal por outros condimentos. A receita modificada obteve redução no seu valor calórico, de lipídios, colesterol, sódio e gordura saturada e aumento no teor protéico e de ácidos graxos poliinsaturados. Essa receita foi avaliada sensorialmente pelo método de escala hedônica, tendo sido aprovada por 78\% dos provadores e identificada como estrogonofe por mais de $90 \%$. Estes resultados mostram que podem ser realizados experimentos com substituições de ingredientes em receitas e com sucesso, a fim de atender às especificidades de cada dieta.

Termos de indexação: colestrol, análise sensorial, dieta com restrição de gorduras, cardiopatias.

\begin{abstract}
ABSTRAC T
Prospective studies of ischemic heart diseases detected risk fators to coronary diseases: age, sex, presence of systemic hypertension, high levels of cholesterol and tabagism. The last three can be modified by dietetic, pharmacologic and/ or behavioral interventions. The capacity of a diet or food in increasing the levels of plasmatic cholesterol and causing atherosclerosis is directly related to its contents of cholesterol and saturated fat. Individuals with a diets restricted on sodium, cholesterol and saturated fat should not consume meat strogonoff, a traditional russian preparation usually eaten by the population, as it includes butter, salt and cream. In order to provide this preparation with adequate nutrients, this study evaluated adaptations such as the substitution of cream by skimmed milk and starch, butter by vegetable oil and salt by spices. In the modified recipe there is a reduction in calories, lipids, cholesterol, sodium and saturated fat, and an increase in
\end{abstract}

(1) Bolsistas do Programa Especial de Treinamento (PET/CAPES), 1995.

(2) Professora Titular do Departamento de Nutrição da Faculdade de Saúde Pública, USP. 
the levels of protein and polyunsaturated fatty acids. This preparation was analyzed by sensory evaluation, using hedonic scale method, and it was accepted by $78 \%$ of the tasters and identified as strogonoff by more than $90 \%$ of them. These results showed that it is possible to make changes in recipes with success, attending to some specific diets.

Index terms: cholesterol, sensory evaluation, diet fat-restricted, heart diseases.

\section{INTRODUÇÃO}

As doenças cardiovasculares constituem a primeira causa de mortes em grande parcela da população adulta, acima de 30 anos de idade, na maioria dos países do Ocidente. Tal posição tem sido mantida, apesar do declínio acentuado da mortalidade observada nos países desenvolvidos, nas últimas décadas. Entre as enfermidades cardiovasculares, a doença isquêmica do coração e as doenças cerebrovasculares são freqüentes (CHOR et al., 1995).

As doenças cardiovasculares têm sido a principal causa de mortes no Brasil desde a década de 60. A doença isquêmica do coração é a principal responsável nas cidades da Região Sul e Sudeste, e a cerebrovascular no Norte-Nordeste e, em ambas as regiões as outras doenças do coração são a terceira causa de mortalidade, principalmente a insuficiência cardíaca (LOLIO et al., 1995).

Em estudos prospectivos, realizados em comunidades, foram identificados os fatores de risco para a doença coronariana tais como: idade, sexo, hipertensão arterial sistêmica, os níveis elevados de colesterol sérico e o tabagismo. Os três últimos merecem interesse especial, uma vez que sua prevalência pode ser modificada por meio de intervenções dietéticas, farmacológicas e/ou comportamentais (SANTOS et al., 1994).

Estudos epidemiológicos têm demonstrado que existe correlação direta entre níveis elevados de colesterol plasmático e aumento de doenças isquêmicas do coração. O potencial de uma dieta ou alimento em aumentar os níveis plasmáticos de colesterol, e promover aterosclerose está diretamente relacionado com seu conteúdo em colesterol e gordura saturada (OLIVEIRA et al., 1991).

Pesquisas realizadas no município de São Paulo, analisando a dieta habitual como fator de risco para doenças cardiovasculares, constataram a prevalência de $38 \%$ de obesidade, $26 \%$ de dislipidemias e $5 \%$ de diabetes. A dieta habitual estava adequada apenas para 5\% da amostra com relação a energia, distribuição calórica dos macronutrientes e quantidade de colesterol (CERVATO, 1995).

O consumo de alimentos com grandes quantidades de ácidos graxos saturados e de colesterol está associado a altos níveis plasmáticos de colesterol e aumento do risco de doenças coronarianas, enquanto que o de ácidos graxos poliinsaturados e monoinsaturados, carboidratos complexos e fibras alimentares estão relacionados com níveis mais baixos de colesterol sanguíneo, determinando baixa incidência dessas doenças (LOLIO et al., 1995).

A intervenção dietética que previne a hipertensão arterial sistêmica é a redução do consumo de sódio, através da restrição do sal adicionado à alimentação. Portanto, mudanças nos hábitos alimentares envolvendo aumento no consumo de peixes e vegetais, substituição do leite integral por leite desnatado, uso de gorduras menos saturadas e óleos poliinsaturados, além da redução do consumo de sal, são medidas que produzem queda nos níveis plasmáticos de colesterol e triglicerídios, controle da pressão arterial, com conseqüente diminuição na incidência das doenças cardiovasculares (OLIVEIRA et al., 1991).

Este trabalho verificou a substituição de ingredientes em preparações com grande quantidade de gordura saturada, o que torna restrito seu consumo em uma dieta para prevenção de doenças cardiovasculares.

A receita escolhida foi o estrogonofe, prato tradicional da cozinha russa (stroganov), conhecido na Europa sob várias formas desde o século XVIII. Consiste numa preparação feita com carne em cubos, coberta com molho cremoso, temperada com cebola 
e cogumelos (LAROUSSE, 1990). Geralmente este molho cremoso é preparado com creme de leite.

Os ingredientes que restrigem o consumo dessa preparação são: manteiga, sal e creme de leite, os quais foram substituídos por ingredientes permitidos em dietas com restrição de sódio, gordura saturada e colesterol, mantendo as características sensoriais semelhantes à tradicional e com padrão de aceitabilidade.

\section{MATERIAL E MÉTODOS}

Este trabalho foi desenvolvido conforme as etapas abaixo relacionadas:

1. Levantamento de receitas existentes de estrogonofe tradicional e de modificadas,

2. Desenvolvimento das receitas em laboratório, utilizando utensílios e equipamentos domésticos, a partir da:

- seleção e caracterização da receita padrão,

- seleção e caracterização de receitas modificadas existentes,

- seleção da receita a ser adaptada,

- adaptação e substituição dos ingredientes não recomendados nesta dieta com restrinções: creme de leite por amido e leite desnatado, sal por condimentos e manteiga por óleo;

- Cálculo da composição centesimal da receita padrão e da modificada pelo programa NUT (Sistema de Apoio à Decisão em Nutrição e Metabolismo da Escola Paulista de Medicina - Departamento de Pediatria);

- Painel de discussão das preparações ao final de cada sessão de laboratório, para identificação do produto final desejado,

- Análise sensorial da preparação modificada para avaliar sua aceitação, através do teste afetivo da escala hedônica, no qual o indivíduo expressa o grau de gostar ou desgostar do produto. Participaram do teste como degustadores, não treinados, 28 alunos do Curso de Nutrição da Faculdade de Saúde Pública da USP. Na ficha de avaliação foi solicitada a identificação do produto degustado, a fim de se confirmar o êxito das modificações realizadas.

\section{RESULTADOS E DISC USSÃO}

Das receitas de estrogonofe tradicionais elaboradas, a selecionada e considerada como padrão foi de preparo mais simplificado (número de ingredientes e utensílios) e que obteve como características do seu molho: cor na tonalidade alaranjada, consistência homogênea de creme e sabor suave característico (Anexo 1).

Uma única receita modificada para pacientes com problemas cardiovasculares foi obtida no levantamento e, ao ser desenvolvida não apresentou as características determinadas como padrão. Essa preparação no final apresentou molho com separação de fases líquida e gordurosa; a cor marrom desagradável e o sabor ácido não característico. Esse molho que utilizou iogurte, não se manteve homogêneo, mesmo observando-se os cuidados na técnica de preparo. Em relação à cor, permaneceu a da carne bovina cozida (marrom claro), não tendo sido amenizada pelos ingredientes presentes, diferentemente do que ocorre com o creme de leite na receita tradicional. A nota de sabor ácido que foi conferida pela presença do iogurte e os condimentos utilizados não foram considerados como característicos do produto estrogonofe. Sendo assim, essa receita não foi utilizada como base para as adaptações propostas, por diferir em muito do padrão estabelecido.

Para a obtenção de uma receita modificada (Anexo 2) com características semelhantes à padrão, considerando cor, consistência e sabor, foram necessárias sete sessões de laboratório.

O creme de leite no estrogonofe, além de conferir sabor ao produto, colabora na textura e cor. A sua substituição na receita tradicional foi feita pelo amido dissolvido em leite desnatado, que tem, na culinária a função de engrossar, gelificar e estabilizar as preparações (GRISWOLD, 1972). O primeiro a ser testado foi o de milho, porém a preparação final não apresentou consistência adequada (elástica e pegajosa), nem cor característica (transparente), e o sabor da preparação ficou prejudicado, pois o amido de milho neutralizou a ação dos temperosutilizados.

A substituição do creme de leite por farinha de trigo e leite desnatado mostrou melhores resultados, porém foram necessários vários testes 
para a determinação da porcentagem adequada a ser utilizada, bem como para a adequação da técnica de preparo (seqüência de adição dos ingredientes). Foram usadas as seguintes concentrações: 26\%, $20 \%, 18 \%, 15 \%$ e $12 \%$. Optou-se por iniciar com quantidades próximas as do creme de leite, as quais foram sendo reduzidas até a obtenção da consistência mais semelhante a da receita padrão (12\%), porém sem anular o sabor dos temperos.

Em relação as técnicas de preparo, fez-se em separado o molho branco, que foi adicionado posteriormente à preparação. Dessa forma, foram obtidos resultados satisfatórios, mas que poderiam ser melhorados. Assim foram realizados outros testes para simplificar o preparo da receita.

Quando a farinha foi adicionada após todos os ingredientes, com exceção do leite, houve a formação de grumos, influenciando na textura final. Obteve-se, então, que o momento ideal de adição da farinha é logo após a cocção da carne, permitindo a dextrinização do amido e garantindo a cremosidade final da preparação.

A substituição do sal por cominho e orégano, como utilizado na receita modificada do Instituto do Coração, e adicionando-se páprica resultou em preparações com sabor não característico de estrogonofe. Optou-se, então, pela utilização dos mesmos condimentos da receita padrão (aumentando a quantidade de cebola), mas com cogumelos frescos do tipo champignon e a inclusão de noz-moscada combinada com uma mistura industrializada de temperos que contém elementos realçadores de sabor. O produto industrializado foi o Sazón vermelho - Ajinomoto (combinação de glutamato monossódico, sal, colorífico, alho, cebola, orégano, louro, salsa e gordura vegetal). Mesmo sendo um produto industrializado, essa mistura contém baixo teor de sódio (14\%). Com essa substituição atingiu-se melhor resultado final do sabor, semelhante ao do estrogonofe padrão. Como o glutamato monossódico (principal componente da mistura Sazón vermelho) tem a propriedade de ressaltar sabores, testou-se separadamente este ingrediente com a noz-moscada. Os resultados obtidos foram semelhantes aos do experimento anterior, apesar de utilizar menor número de condimentos, mas apresentou sabor menos intenso e ficou ligeiramente mais claro. Tendo em vista o resultado e a semelhança com a receita considerada padrão, a preparação com a mistura industrializada foi considerada como final para avaliação no teste de aceitação.

A receita modificada foi submetida a análise sensorial e os resultados obtidos são apresentados na Figura 1. Observa-se que $78,6 \%$ das pessoas apresentaram aceitação acima de gostei ligeiramente (valor 6 na escala hedônica), ou seja, a preparação foi aprovada pela maioria dos degustadores. Na ficha de avaliação, 92,8\% anotaram como sendo estrogonofe no item identificação, mostrando o êxito das modificações realizadas.

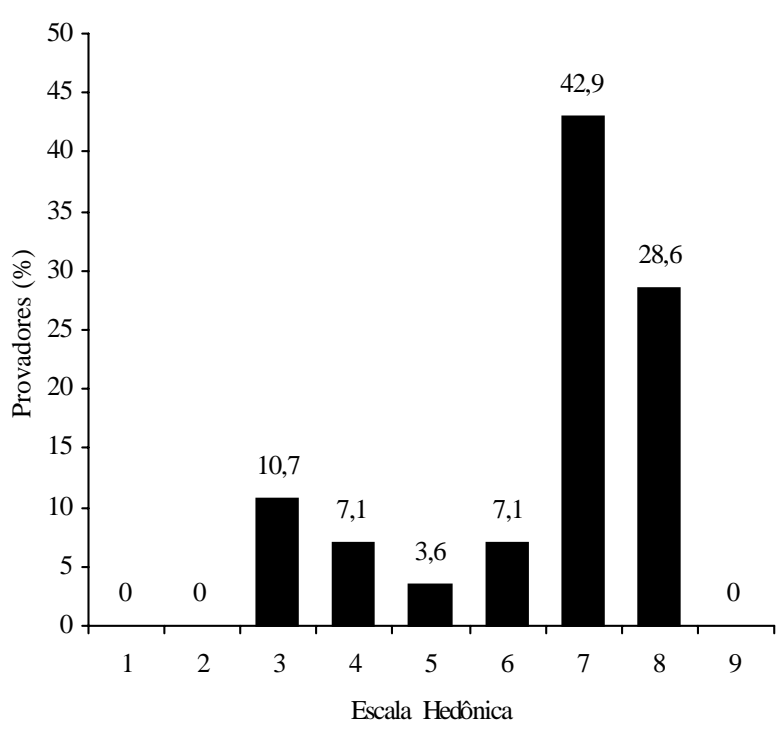

Figura 1. Distribuição dos provadores (\%) em função dos valores hedônicos marcados na escala para o estrogonofe modificado.

Na Tabela 1 é apresentada a comparação de duas receitas de estrogonofe, padrão e modificada, em relação a alguns nutrientes. Observase que houve redução de $25,0 \%$ no valor calórico, $58,6 \%$ no total de lipídios, $65,3 \%$ de sódio, $77,7 \%$ de ácidos graxos saturados e 34,0\% de colesterol em relação à preparação original.

A diferença constatada na composição foi importante, mostrando a possibilidade de se incluir essa preparação em dietas especiais, como variação nos cardápios. A redução de 65,3\% no teor de sódio 
Tabela 1. Composição (em 100g) do estrogonofe padrão e do modificado, em calorias e alguns nutrientes.

\begin{tabular}{lcc}
\hline Nutrientes & Padrão & Modificado \\
\hline Proteína(g) & 14,80 & 18,62 \\
Lipídios (g) & 18,53 & 7,68 \\
Carboidratos (g) & 3,08 & 8,34 \\
Sódio (mg)* & $328,60(14 \mathrm{mEq})$ & $114,25(4,9 \mathrm{mEq})$ \\
Ácidos graxos saturados (g) & 9,60 & 2,15 \\
Ácidos graxos poliinsaturados (g) & 0,35 & 1,44 \\
Colesterol (mg) & 81,00 & 53,47 \\
Calorias (kcal) & 241,06 & 182,87 \\
\hline
\end{tabular}

*O teor de sódio do Sazon foi fornecido pela Ajinomoto Interamericana.

proporciona maior flexibilidade no cardápio, permitindo a inclusão deste mineral em outras preparações apreciadas pelo paciente. Em relação aos lipídios e, em especial, aos ácidos graxos saturados, a preparação modificada apresentou valores bem inferiores, fato este imprescindível na dieta de pacientes com restrição alimentar desses nutrientes.

O fato da preparação ter sido bem aceita por uma população de degustadores composta de indivíduos saudáveis, representa bom indicador como variação em dietas para aqueles que têm restrições alimentares. Na falta do condimento Sázon vermelho pode ser utilizado, com bons resultados, o glutamato monossódico acrescido de folha de louro, orégano e salsa.

\section{CONCLUSÃO}

A receita adaptada apresentou redução de $25 \%$ no valor calórico, 58,6\% no total de lipídios, $65,3 \%$ de sódio, $77,7 \%$ de ácidos graxos saturados e 34,0\% de colesterol, aumento no teor protéico e em ácidos graxos poliinsaturados em relação a receita original, o que viabiliza a utilização do estrogonofe com vantagens em dietas com estas restrições, garantindo variação das preparações.

A utilização de farinha de trigo e leite desnatado supriu as características da textura do estrogonofe, bem como colaborou com a combinação dos condimentos para o sabor da preparação.

A combinação de condimentos ricos em glutamato monossódico intensificou a ação do conjunto de ingredientes responsáveis pelo sabor.

A análise sensorial mostrou que houve aprovação da preparação por $78,6 \%$ da população testada, que assinalou na escala hedônica valores acima de 6 (de gostei ligeiramente a gostei muito).
No que se refere à identificação da preparação, na ficha de avaliação, 92,8\% anotaram como sendo estrogonofe, mostrando o êxito das modificações realizadas.

O conhecimento das funções dos ingredientes e a caracterização das preparações são fundamentais para o sucesso na substituição dos ingredientes.

A variação nos cardápios é importante para qualquer paciente que tenha restrição em sua dieta, pois a alimentação em nossa sociedade é fator de extrema relevância, momento de participação famíliar, de satisfação não somente orgânica mas também psicológica. Sabendo da possibilidade da variação em determinadas ocasiões, o seguimento da dieta torna-se mais fiel e a recuperação do indivíduo mais rápida.

Estudos com outras preparações devem ser realizados, utilizando ingredientes de fácil acesso, simplicidade na preparação e sempre levando em consideração o modo de vida do indivíduo e suas preferências.

\section{REFERÊNC IASBIBLIO GRÁFIC AS}

CERVATO, A.M. Dieta habitual e fatores de risco para doenças cardiovasculares. São Paulo, 1995. 84p. Dissertação (Mestrado) - Faculdade de Saúde Pública, USP, 1995.

CHOR, D., FONSECA, M.J.M., ANDRADE, C. R. Doenças cardiovasculares: comentários sobre mortalidade precoce no Brasil. Arquivos Brasileiros de Cardiologia, São Paulo, v.64, n.1, p.15-19, 1995.

GRISWOLD, R.M.N. Estudo experimental dos alimentos. São Paulo : Edgard Blucher, 1972. 469p. 
LAROUSSE. Gastronomique: the world's greatest cookery encyclopedia. Londres : Mandarin Paperbacks, 1990. p.1233-1234.

LOLIO, C.A., LOTUFO, P.A., LIRA, A.C., ZANETTA, D.M.T., MASSAD, E. Tendência da mortalidade por doença isquêmica do coração nas capitais de regiões metropolitanas do Brasil, 1979-89. Arquivos Brasileiros de Cardiologia, São Paulo, v.64, n.3, p.213-216, 1995.

OLIVEIRA, S.P., TAHIN, Q.S., CAVALCANTI, T.C. Epidemiologia das doenças isquêmicas do coração: papel da dieta. Revista de Nutrição da PUCCAMP, Campinas, v. 4, n.1, p.146-153, 1991.

SANTOS, J.E., DRESSLER,W.W., VITERI, F. Fatores de risco da doença arterial coronariana e suas correlações com variáveis dietéticas e sociais. Arquivos Brasileiros de Cardiologia, São Paulo, v.63, n.5, p.371-375, 1994.

Recebido para publicação em 4 de junho de 1996 e aceito em 10 de junho de 1997. 
ANEXO 1

RECEITA PADRÃO

\section{Ingredientes}

1 Kg de filé mignon

sal a gosto

2 cebolas (médias) picadas

5 colheres (sopa) de extrato de tomate

2 colheres (sopa) de catchup

1 colher (sopa) de mostarda

$200 \mathrm{~g}$ de cogumelo tipo champignon

1 lata de creme de leite

\section{Preparo}

Cortar a carne em cubos

Temperar a carne com sal;

Derreter 3 colheres (sopa) de manteiga e fritar a carne;

Tirar a carne da panela reservar;

Derreter 2 colheres (sopa) de manteiga e dourar a cebola;

Colocar a carne na panela e, em seguida, adicionar o extrato de tomate, o catchup, a mostarda e os cogumelos;

Adicionar o creme de leite e aquecer por alguns minutos.

\section{ANEXO 2}

REC EITA MODIFICADA

\section{Ingredientes}

1 Kg de carne (alcatra ou coxão mole)

2 envelopes de "Sazon vermelho" (10g)

2 cebolas (médias) picadas

8 colheres (sopa) de farinha de trigo

3 colheres (sopa) de extrato de tomate

2 colheres (sopa) de catchup

1 colher (sopa) de mostarda

$200 \mathrm{~g}$ de cogumelos frescos tipo champignon

noz moscada q.s.

4 xícaras de leite desnatado

8 colheres (sopa) de óleo vegetal (soja, milho, etc.)

Preparo

Remover toda a gordura existente na carne e cortar em cubos;

Temperar a carne com "Sazon vermelho";

Colocar 6 colheres (sopa) de óleo na panela e fritar a carne;

Tirar a carne da panela e reservar;

Colocar 2 colheres (sopa) de óleo e dourar a cebola;

Colocar a carne na panela e, em seguida, adicionar a farinha de trigo e mexer sempre até dourá-la;

Adicionar o extrato de tomate, o catchup, a mostarda, os cogumelos e a noz moscada;

Adicionar o leite, aos poucos, mexendo sempre. 